Le Coenothele gregalis Simon a pu vivre à Paris dans une pièce tantôt fermée, tantòt ouverte pendant la nuit, depuis la fin d'avril jusqu'au milieu d'août. A cette date, deux individus seulement étaient parvenus à l'état adulte. Ils présentaient alors divers traits de mœurs déjà signalés par L. Diguet chez ceux du Mexique. Il n'était plus nécessaire de les approvisionner de mouches. lls se nourrissaient de celles qui se prenaient dans la toile. Ces faits font espérer la réussite d'une nouvelle tentative d'acclimatement.

Quoi qu'il en soit, celle de 1910 aura eu l'intérêt de montrer comment les araignées ont constitué leur toile à différents âges et comment leur nid réalise, dans sont état final, un type jusqu'ici inusité.

\title{
Description d'un Lucanide nouveau [COL.]
}

par H. Bolleau.

Metallactulus Bennigseni, n. sp. - Insecte de taille inférieure à celle du $\boldsymbol{M}$. parvulus $\mathrm{Hope,} \mathrm{ayant} \mathrm{la} \mathrm{même} \mathrm{apparence} \mathrm{courte} \mathrm{et}$ cylindrique, mais facile à distinguer de cette espèce par la forme des mandibules, la couleur, et le poli des élytres.

๙. Les mandibules sont un peu plus longues, plus régulièrement cintrées et plus grêles que celles du $\boldsymbol{M}$. parvulus; elles sont sensiblement plus longues que la tête chez les grands exemplaires. L'extrémité se termine par une fourche dont la pointe inférieure est plus longue et plus aiguë que la supérieure. Il existe une dent simple, aiguë, relevée vers le haut, un peu plus près de la base que de la pointe; cette dent, chez $\boldsymbol{M}$. parvulus, est horizontale et émoussée. De plus, chez M. Bennigseni, on voit sur l'arête inférieure interne de la mandibule, deux très petites dents, au-dessous de la dent médiane principale, qui est placée entre elles mais sur l'arête supérieure.

La tête est convexe; la partie frontale régulièrement bombée, les deux tubercules du M. parvulus, à peine indiqués, sont difficilement visibles chez certains exemplaires. La lèvre supérieure, très transversale, est à peine convexe, entièrement différente de la forme en accolade de celle de $\boldsymbol{M}$. parvulus. Les angles antérieurs de la tête sont très arrondis; ils se continuent presque directement par les canthus, dont le contour est régulièrement convexe et qui s'avancent à peu près jusqu'à la moitié de l'œil, en diminuant rapidement de largeur. 
Les antennes ressemblent beaucoup à celles du $\boldsymbol{M}$. parvulus, mais ont les lamelles du peigne moins allongées. Le peigne est formé par les trois derniers articles; le huitième n'est pas spongieux sur la face opposée à la lamelle, les deux derniers ont leur surface entièrement spongieuse. Les six articles qui précèdent le peigne, ainsi que la face non spongieuse du huitième article, sont ternes et dépolis, le scape et le deuxième article étant, comme chez $\boldsymbol{M}$. parvulus, seuls brillants. Le troisième article est un peu plus long que le quatrième; celuici et les suivants vont en augmentant progressivement, le sixième article est un peu renflé, le septième est plus nettement anguleux, sans toutefois former un éperon ou un prolongement digitiforme. Chez M. parvulus le septième article est anguleux et le sixième est de la même forme que les précédents.

Le menton est large, très fortement arrondi en avant, presque semi-elliptique. Sa surface est dépolie et très finement ponctuée.

Le prothorax est régulièrement convexe, finement bordé sur la marge antérieure; les angles latéraux antérieurs, assez avancés,

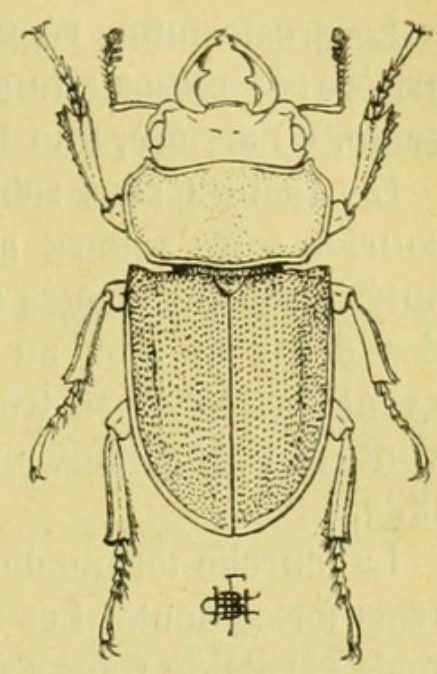

Fig. 1. - Vetallactulus Bennigseni Boileau. emboitent étroitement la tète et présentent un contour assez compliqué formé, en avant, par l'are concave du bord antérieur auquel succède une ligne oblique convexe qui rencontre sous un angle aigu le contour latéral. Celui-ci est constitué par un arc concave extérieurement, raccordé lui-mème à l'arc faiblement convexe qui forme le còté du prothorax. Le tout rappelle un peu ce que l'on voit chez l'Hemisodorcus elegantulus Albers. Les còtés sont presque parallèles; l'angle médian est bien marqué, mais non épineux; il en est de mème pour l'angle postérieur. Entre ces angles, le contour est légèrement concave; le bord postérieur est faiblement convexe, à peine un peu sinueux.

L'écusson est ogival, arrondi aux angles.

Les élytres sont un peu plus allongés que chez parvulus, leur base est un peu oblique avec l'angle huméral légèrement épineux, leur contour est régulièrement convexe.

Toute la surface supérieure est brillante; la tète et le prothorax sont cependant très finement dépolis; les élytres sont plus brillants. Le pronotum porte une ponctuation fine et espacée, visible seulement à la loupe; les élytres présentent une ponctuation plus_distincte des- 
sinant, outre la ligne suturale, trois ou quatre autres intervalles étroits séparés par des espaces ponctués plus larges. Cette disposition est d'ailleurs peu visible, les points, quoique bien marqués, étant très fins.

En dessous, les pièces sternales sont brillantes, ponctuées sur les còtés, lisses sur la région centrale; les épisternes et les hanches sont ponctués, les segments abdominaux sont faiblement ponctués le long de leur bord postérieur.

Le prosternum, régulièrement arrondi, ne forme aucune saillie en arrière; le mésosternum êst également convexe; le métasternum présente, en arrière, une légère dépression longitudinale médiane.

Les fémurs sont robustes et portent, en dessous, deux lignes de courtes soies raides, asséz écartées les unes des autres. Les tibias antérieurs sont armés extérieurement de dents inclinées analogues à des dents de scie, au nombre de deux ou trois, entre lesquelles et avant lesquelles se trouvent d'autres denticules de même forme qui vont en augmentant un peu, comme dimensions, vers l'extrémité du tibia.

La fourche terminale habituelle est ici remplacée par un groupe de trois fortes dents. Les tibias médians et les postérieurs sont inermes, mais garnis de soies raides et courtes, qui existent aussi, moins nombreuses, sur le bord denté des tibias antérieurs.

Les tarses sont un peu plus courts que les tibias, l'article terminal est sensiblement aussi long que trois autres articles pris ensemble. Les quatre premiers articles sont revêtus inférieurement de soies plus longues à l'extrémité de chaque article qu'a sa naissance.

Les petits màles diffèrent très peu des grands. Leurs mandibules sont plus courtes, mais conservent le mème nombre de dents, et celles-ci ne paraissent pas avoir de tendance a diminuer d'importance. Les tibias médians et postérieurs restent inermes.

†. Les femelles sont aussi très peu différentes des mâles; leur ponctuation est cependant plus forte sur la tête et les pièces thoraciques.

La tète est bombée, brillante, avec une ponctuation assez forte, plus serrée sur la région frontale et les joues. Il existe, vers le sommet de la tête, à l'endroit oủ l'on vioit deux petites protubérances chez M. parvulus $\&$, une sorte de croissant lisse formant une faible saillie sur le reste de la surface.

Les contours antérieurs de la tête sont très analogues à ceux du mâle, mais la lèvre supérieure, beaucoup moins large, est légèrement échancrée en avant. Le menton, en demi-ellipse, est couvert de gros points enfoncés.

Les mandibules sont assez longues, fortement carénées; leur pointe 
terminale est simple; il existe une dent médiane placée sur l'arête inférieure interne; cette dent, peu aigüe, se prolonge vers la base par une carène legèrement concave dont l'extrémité forme une deuxième dent, peu saillante.

Les antennes sont un peu plus courtes que chez le mâle. Les tibias antérieurs ont même armature, mais sont plus larges. Les médians et les postérieurs sont rugueux mais sans épines.

La cọloration est la même dans les deux sexes. La tête et le prothorax sont d'un brun noir très foncé; les élytres d'une nuance un peu moins sombre et plus rougeâtre; la partie inférieure est sensiblement de la même couleur que les élytres. Les pattes sont rougeâtres.

Les dimensions principales sont :

Longueur totale, mandibules incluses : o 11 mill. 6 à 14 mill. 5, † 13 mill. ä a 15 mill. 2.

Long. des mandibules : ơ 1 mill. 5 à 3 mill., o 1 mill. 4 à 1 mill. 7 . Longueur des élytres : $0^{-1} 6$ mill. 8 à 8 mill. 4, of 7 mill. 8 à 9 mill. Largeur maxima, au prothorax : đ 4 mill. 8 a $̋$ mill. 8 , o ว̆ mill. 7 i 6 mill. 2.

Plusieurs spécimens des deux sexes, provenant de Ponape (illes Carolines).

\section{Description de deux Lucioles nouvelles de la Nouvelle-Guinée}

[CoL. LAMPYRIDAE]

par Ernest Olivier.

M. L. von Hexdes m'a communiqué deux Lucioles faisant partie des collectionś du Musée Senckenberg, à Francfort-sur-le-Main, qui ont été capturées par le $\mathrm{D}^{\mathrm{r}}$ Wolf dans l'archipel de la Nouvelle-Guinée. Ces deux Lucioles constituent des espèces nouvelles bien caractérisées dont je donne ici les descriptions. Il n'y a malheureusement de chacune qu'un seul individu femelle.

Luciola Wolfi, n. sp. - Oblonga, ampla, convexa, dilute flava, oculis nigris, antennis et tarsis piceis, fronte macuia parva fusca notata; elytris fuscis, sutura tenuiter et margine externo latius flavo limbatis; prothorace brevi, semilunari, punctato, in medio longitudinaliter sulcatulo, margine antico valde erecto, basi recte truncato, angulis 


\section{$2 \mathrm{BHL}$ Biodiversity Heritage Library}

1911. "Description d'un Lucanide nouveau." Bulletin de la Société entomologique de France 1910, 340-343. https://doi.org/10.5962/bhl.part.4696.

View This Item Online: https://www.biodiversitylibrary.org/item/38239

DOI: https://doi.org/10.5962/bhl.part.4696

Permalink: https://www.biodiversitylibrary.org/partpdf/4696

\section{Holding Institution}

Smithsonian Libraries

\section{Sponsored by}

Smithsonian

\section{Copyright \& Reuse}

Copyright Status: NOT_IN_COPYRIGHT

This document was created from content at the Biodiversity Heritage Library, the world's largest open access digital library for biodiversity literature and archives. Visit BHL at https://www.biodiversitylibrary.org. 